\section{Adaptive Array Beamforming with Robust Capabilities Under Random Phase Perturbations}

\author{
Ju-Hong Lee and Kuang-Peng Cheng
}

\begin{abstract}
The problem of adaptive array beamforming with multiplebeam constraints in the presence of steering error caused by random phase perturbations is considered. We first construct a cost function consisting of terms that utilize a posteriori information due to the received array data and $a$ priori information due to the probabilistic distribution of phase perturbation, respectively. Then, an appropriate estimate of the actual phase angle vector associated with each of the desired signals can be obtained by performing a nonlinear optimization problem based on the cost function. An implementation algorithm is further presented to iteratively solve the problem. Theoretical analysis regarding the convergence property of the iterative procedure is also investigated. Finally, several computer simulation examples are provided for illustration.
\end{abstract}

Index Terms-Adaptive array beamforming, random phase perturbations, steering error.

\section{INTRODUCTION}

An adaptive array beamformer is designed for automatically preserving the desired signals while canceling the interference and noise. The only a priori knowledge for a main-beam or a multiple-beam constrained beamformer is the actual direction vectors of the desired signals. A direction vector of a desired signal can be obtained from the information of the array sensor locations, signal impinging directions, and the propagation characteristics. However, the information may not be perfectly known in practice. This results in a mismatch between the presumed steering vectors and the actual direction vectors. The performance of a steered beam adaptive array beamformer is very sensitive to the mismatch [1]-[5].

To cure the problem due to the above mismatch, most robust techniques propose to impose additional constraints such as multiple linear constraints, derivative constraints, and norm constraints on the array weight vector [5]-[15]. However, imposing additional constraints deteriorates the array capability in suppressing interference and noise. In contrast, Shahbazpanahi et al. [16] present a robust approach based on the worst-case performance optimization for curing the problem of array performance degradation due to the signal covariance matrix with some fixed error. Li et al. [17] propose a diagonal loading approach for the beamforming problem of the desired signal with nonrandom steering vector error. Recently, based on the assumption that the steering vector error is an additive Gaussian random vector, two methods have been presented in [18] to find two appropriate closedform solutions for estimating the optimal steering constraint vector. All of the above-mentioned techniques [5]-[18] are developed under the situation of adaptive beamforming with main-beam constraint. In many applications, such as satellite communications [19], an antenna array must possess beamforming capability to receive more than one signal with specified gain requirements while suppressing all jammers. This purpose can be effectively achieved by using an antenna array

Manuscript received June 13, 2003; revised January 21, 2004. This work was supported by the National Science Council under Grant NSC90-2213-E002043. The associate editor coordinating the review of this paper and approving it for publication was Dr. Martin Haardt.

The authors are with the Department of Electrical Engineering, National Taiwan University, Taipei 10617, Taiwan, R.O.C. (e-mail: juhong@ cc.ee.ntu.edu.tw).

Digital Object Identifier 10.1109/TSP.2004.838961 with multiple-beam pattern [19], [20]. Recently, a technique for adaptive beamforming with the capability of providing multiple-beam constraints (MBCs) has been presented in [21].

In this correspondence, we consider the problem of adaptive beamforming with $\mathrm{MBC}$ in the presence of random phase perturbations. A robust method in conjunction with an iterative procedure is presented for solving the considered problem. To find the optimal phase angle vector, we construct a cost function consisting of the squared norm of the projection of the steering vector on the noise subspace and a constraint related to a likelihood function associated with the random phase error vector. Minimizing the squared norm of the projection of the steering vector on the noise subspace is equivalent to maximizing the squared norm of the projection of the steering vector on the signal plus interference subspace. The constraint related to a likelihood function associated with the random phase error vector is utilized to prevent the obtained optimal phase angle vector for each desired signal from becoming one of the interference phase angle vectors. Since the resulting minimization problem is highly nonlinear, we use a gradient method to iteratively search for the solution. It is shown that using the constraint related to a likelihood function of the random phase error vector provides the advantage of properly adjusting the step size during the gradient search procedure. The analysis of the convergence property of the proposed method is also presented. Several computer simulation examples show the effectiveness of the proposed method.

\section{PROBLEM Formulation}

Consider a uniform linear array (ULA) with $M$ sensors and interelement spacing equal to half of the smallest wavelength of the signals with specified gain/null arrangements. Let $K$ narrowband and far-field signals be impinging on the array from direction angles $\theta_{i}, i=1,2, \ldots, K$, off broadside. The signal received at the $m$ th array sensor can be expressed as

$$
x_{m}(t)=\sum_{i=1}^{K} s_{i}(t) a_{m}\left(\theta_{i}\right)+n_{m}(t), \quad m=1,2, \ldots M
$$

where $a_{m}\left(\theta_{i}\right)=\exp \left(\mathrm{j}\left(2 \pi d_{m} \sin \theta_{i}\right) / \lambda_{i}\right), \lambda_{i}$ is the wavelength of the $i$ th signal, and $d_{m}$ is the distance between the $m$ th and the first array sensors, $s_{i}(\mathrm{t})$ is the complex waveform of the $i$ th signal, and $n_{m}(\mathrm{t})$ is the spatially white noise with mean zero and variance $\sigma_{n}^{2}$ received at the $m$ th array sensor. In matrix form, we can write the data vector received by the ULA as follows:

$$
x(\mathrm{t})=\boldsymbol{B} \boldsymbol{s}(\mathrm{t})+\boldsymbol{n}(\mathrm{t})
$$

where the matrix $\boldsymbol{B}=\left[\begin{array}{llll}\boldsymbol{a}\left(\theta_{1}\right) & \boldsymbol{a}\left(\theta_{2}\right) & \ldots & \boldsymbol{a}\left(\theta_{K}\right)\end{array}\right]$ with the direction vector of the $i$ th signal given by $\boldsymbol{a}\left(\theta_{i}\right)=\left[\begin{array}{llll}a_{1}\left(\theta_{i}\right) & a_{2}\left(\theta_{i}\right) & \ldots & a_{M}\left(\theta_{i}\right)\end{array}\right]^{\mathrm{T}}$, the signal source vector is $\boldsymbol{s}(\mathrm{t})=\left[\begin{array}{lllll}s_{1}(\mathrm{t}) & s_{2}(\mathrm{t}) & \ldots & s_{K}(\mathrm{t})\end{array}\right]^{\mathrm{T}}$, and the noise vector is $\boldsymbol{n}(\mathrm{t})=\left[\begin{array}{llll}n_{1}(\mathrm{t}) & n_{2}(\mathrm{t}) & \ldots & n_{M}(\mathrm{t})\end{array}\right]^{\mathrm{T}}$. The superscript $\mathrm{T}$ denotes transpose operation. Under the assumption that $\boldsymbol{s}(\mathrm{t})$ and $\boldsymbol{n}(\mathrm{t})$ are uncorrelated, the $M \times M$ ensemble correlation matrix of $\boldsymbol{x}(\mathrm{t})$ is Toeplitz-Hermitian and given by

$$
\boldsymbol{R}_{x}=\left[R_{k l}\right]=[R(k-l)]=\mathrm{E}\left\{\boldsymbol{x}(\mathrm{t}) \boldsymbol{x}(\mathrm{t})^{\mathrm{H}}\right\}=\boldsymbol{B} \boldsymbol{R}_{s} \boldsymbol{B}^{\mathrm{H}}+\sigma_{n}^{2} \boldsymbol{I}
$$

where the superscript $\mathrm{H}$ denotes the complex conjugate transpose. $\boldsymbol{R}_{s}=\mathrm{E}\left\{\boldsymbol{s}(\mathrm{t}) \boldsymbol{s}(\mathrm{t})^{\mathrm{H}}\right\}$ has rank $K$ if the $K$ signals are uncorrelated. Let the ULA use a weight vector $\boldsymbol{w}=\left[\begin{array}{llll}w_{1} & w_{2} & \ldots & w_{M}\end{array}\right]$ for processing the received data vector $\boldsymbol{x}(\mathrm{t})$ to produce the array output signal 
$\mathrm{y}(\mathrm{t})=\boldsymbol{w}^{\mathrm{H}} \boldsymbol{x}(\mathrm{t})$. Assume that the selective gain/null requirements are specified by assigning a gain $c_{p}$ at the direction vector $\boldsymbol{a}\left(\theta_{p}\right)$ for $p=1,2, \ldots, P$, where $P$ denotes the number of signals with gain/null constraint. Then, the optimal weight vector for the adaptive array can be found from the following constrained optimization problem [19]:

$$
\text { Minimize } \mathrm{E}\left\{|\mathrm{y}(\mathrm{t})|^{2}\right\}=\boldsymbol{w}^{\mathrm{H}} \boldsymbol{R}_{x} \boldsymbol{w} \quad \text { Subject to } G^{\mathrm{H}} \boldsymbol{w}=c
$$

where the matrix $\boldsymbol{G}=\left[\begin{array}{llll}\boldsymbol{a}\left(\theta_{1}\right) & \boldsymbol{a}\left(\theta_{2}\right) \ldots \boldsymbol{a}\left(\theta_{P}\right)\end{array}\right]$ denotes the constraint matrix, and $c=\left[\begin{array}{lllll}c_{1} & c_{2} & \ldots & c_{P}\end{array}\right]$ denotes the gain vector. Accordingly, the optimal weight vector is given by

$$
\boldsymbol{w}_{o}=\boldsymbol{R}_{x}^{-1} \boldsymbol{G}\left(\boldsymbol{G}^{\mathrm{H}} \boldsymbol{R}_{x}^{-1} \boldsymbol{G}\right)^{-1} \boldsymbol{c} .
$$

Substituting (5) into $\mathrm{E}\left\{|\mathrm{y}(\mathrm{t})|^{2}\right\}=\boldsymbol{w}^{\mathrm{H}} \boldsymbol{R}_{x} \boldsymbol{w}$ yields the corresponding array output power equal to

$$
\mathrm{E}\left\{|\mathrm{y}(\mathrm{t})|^{2}\right\}=\boldsymbol{w}^{\mathrm{H}} \boldsymbol{R}_{x} \boldsymbol{w}=\boldsymbol{c}^{\mathrm{H}}\left(\boldsymbol{G}^{\mathrm{H}} \boldsymbol{R}_{x}^{-1} \boldsymbol{G}\right)^{-1} \boldsymbol{c} .
$$

In the presence of random phase perturbation, we consider that the phase angle error vector for the signal with gain $c_{p}$ due to an additive perturbation is given by

$$
\Theta_{e p}=\Theta_{p}-\Theta_{d p}
$$

where $\Theta_{p}$ and $\Theta_{d p}$ denote the phase angle vectors associated with actual direction vector $\boldsymbol{a}\left(\theta_{p}\right)$ and the presumed direction vector $\boldsymbol{a}_{d}\left(\theta_{p}\right)$, respectively. Without loss of generality, let the $m$ th entry of the direction vector $\boldsymbol{a}\left(\theta_{p}\right)$ be expressed as $a_{m}\left(\theta_{p}\right)=\exp \left(\mathrm{j} v_{p m}\right)$ and the corresponding phase angle vector be constructed as $\boldsymbol{\Theta}_{p}=\left[\begin{array}{llll}v_{p 1} & v_{p 2} & \ldots & v_{p M}\end{array}\right]^{\mathrm{T}}$. Similarly, let the $m$ th entry of the direction vector $\boldsymbol{a}_{d}\left(\theta_{p}\right)$ be expressed as $a_{d m}\left(\theta_{p}\right)=\exp \left(\mathrm{j} v_{d p m}\right)$ and the phase angle vector be constructed as $\Theta_{d p}=\left[\begin{array}{llll}v_{d p 1} & v_{d p 2} & \ldots & v_{d p M}\end{array}\right]^{\mathrm{T}}$. According to the discussion of [5], the phase angle error vector $\Theta_{e p}$ shown by (7) can be assumed to be a real Gaussian random vector with mean zero and covariance matrix $D_{p}$. Based on this discussion, we consider the case of Gaussian random phase perturbation. Hence, the probability density function (PDF) for $\Theta_{e p}$ is given by

$$
\operatorname{PDF}\left(\boldsymbol{\Theta}_{e p}\right)=\left[(2 \pi)^{\mathrm{M}} \operatorname{det}\left(\boldsymbol{D}_{p}\right)\right]^{-1 / 2} \exp \left\{-\left(\boldsymbol{\Theta}_{e p}^{\mathrm{T}} \boldsymbol{D}_{p}^{-1} \boldsymbol{\Theta}_{e p}\right) / 2\right\} .
$$

Accordingly, the likelihood function (LF) regarding the phase angle error vector can be defined as

$$
\begin{aligned}
\mathrm{LF} & =\exp \left\{-\left(\boldsymbol{\Theta}_{e p}^{\mathrm{T}} \boldsymbol{D}_{p}^{-1} \boldsymbol{\Theta}_{e p}\right) / 2\right\} \\
& =\exp \left\{-\left[\left(\boldsymbol{\Theta}_{p}-\boldsymbol{\Theta}_{d p}\right)^{\mathrm{T}} \boldsymbol{D}_{p}^{-1}\left(\boldsymbol{\Theta}_{p}-\boldsymbol{\Theta}_{d p}\right)\right] / 2\right\} .
\end{aligned}
$$

Next, consider the case that the $P$ signals with gain/null constraint are uncorrelated and the $P$ phase angle error vectors $\Theta_{e p}, p=1,2, \ldots, P$ are also uncorrelated. We construct an $M \times P$ phase angle error matrix $\Psi$ and its vectorized version $\boldsymbol{\Gamma}$ with size $\mathrm{PM} \times 1$ for the $P$ uncorrelated signals as follows. $\boldsymbol{\Psi}=\left[\begin{array}{llll}\boldsymbol{\Theta}_{e 1} & \boldsymbol{\Theta}_{e 2} & \ldots & \boldsymbol{\Theta}_{e P}\end{array}\right]$ and $\boldsymbol{\Gamma}=\left[\begin{array}{llll}\boldsymbol{\Theta}_{e 1}^{\mathrm{T}} & \boldsymbol{\Theta}_{e 2}^{\mathrm{T}} & \ldots & \boldsymbol{\Theta}_{e P}^{\mathrm{T}}\end{array}\right]^{\mathrm{T}}$. The joint PDF for $\Psi$ can be expressed as

$$
\operatorname{PDF}(\boldsymbol{\Psi})=\left\{\prod_{p=1}^{P}\left[(2 \pi)^{\mathrm{M}} \operatorname{det}\left(\boldsymbol{D}_{p}\right)\right]^{-1 / 2}\right\} \exp \left\{-\left(\boldsymbol{\Gamma}^{\mathrm{T}} \boldsymbol{\Omega}^{-1} \boldsymbol{\Gamma}\right) / 2\right\}
$$

where $\Omega$ is a $\mathrm{PM} \times \mathrm{PM}$ block diagonal matrix with the $p$ th $M \times M$ diagonal block matrix given by $\boldsymbol{D}_{p}$. The corresponding likelihood function regarding the phase angle error vector $\Gamma$ is given by

$$
\begin{aligned}
\mathrm{LF}_{P} & =\exp \left\{-\left(\boldsymbol{\Gamma}^{\mathrm{T}} \boldsymbol{\Omega}^{-1} \boldsymbol{\Gamma}\right) / 2\right\} \\
& =\exp \left\{-\sum_{p=1}^{P}\left[\left(\boldsymbol{\Theta}_{p}-\boldsymbol{\Theta}_{d p}\right)^{\mathrm{T}} \boldsymbol{D}_{p}^{-1}\left(\boldsymbol{\Theta}_{p}-\boldsymbol{\Theta}_{d p}\right)\right] / 2\right\}
\end{aligned}
$$

To deal with the problem of array beamforming with MBC in the presence of phase angle errors, as described above, we present a robust method based on the likelihood function given by (11).

\section{RoBust METHOD}

From the property of a gain constrained array beamformer, it is well known that the output power of the beamformer will achieve its maximum when each presumed direction vector $\boldsymbol{a}_{d}\left(\theta_{p}\right)$ of the constraint matrix $G$ coincides with the actual direction vector $\boldsymbol{a}\left(\theta_{p}\right), p=1,2, \ldots, P$. Moreover, from the eigendecomposition of $\boldsymbol{R}_{x}$ [22], we can express $\boldsymbol{R}_{x}=\sum_{i=1}^{M} \lambda_{i} \boldsymbol{e}_{i} \boldsymbol{e}_{i}^{\mathrm{H}}$, where $\lambda_{1} \geqq \lambda_{2} \geqq \cdots \geqq \lambda_{J+P} \geqq \lambda_{J+P+1}=\ldots=\lambda_{M}=\sigma_{n}^{2}$ are the eigenvalues of $\boldsymbol{R}_{x}$ in the descending order, and $e_{i}$ are the corresponding eigenvectors. $J$ is the number of interferers. The eigenvectors associated with the minimum eigenvalue $\sigma_{n}^{2}$ are orthogonal to the direction vectors of the signals with specified gain/null constraints and interferers. Therefore, the subspaces spanned by $\boldsymbol{E}_{n}=\left\{\boldsymbol{e}_{J+P+1}, \ldots, \boldsymbol{e}_{M}\right\}$ (called the noise subspace) and $\boldsymbol{E}_{s}=\left\{\boldsymbol{e}_{1}, \boldsymbol{e}_{2}, \ldots, \boldsymbol{e}_{J+P}\right\}$ (called the signal plus interference subspace) are orthogonal. We can rewrite $\boldsymbol{R}_{x}$ as follows:

$$
\boldsymbol{R}_{x}=\sum_{i=1}^{M} \lambda_{i} \boldsymbol{e}_{i} e_{i}^{H}=\boldsymbol{E}_{s} \boldsymbol{\Lambda}_{s} \boldsymbol{E}_{s}^{\mathrm{H}}+\boldsymbol{E}_{n} \boldsymbol{\Lambda}_{n} \boldsymbol{E}_{n}^{\mathrm{H}}
$$

where $\boldsymbol{\Lambda}_{s}=\operatorname{diag}\left\{\lambda_{1}, \lambda_{2}, \ldots, \lambda_{J+P}\right\}$, and $\boldsymbol{\Lambda}_{n}=\sigma_{n}^{2} \boldsymbol{I}$, where $\boldsymbol{I}$ denotes the identity matrix with appropriate size. Based on (6), (11), and (12), an appropriate cost function regarding the phase angle errors is defined as

$$
\begin{aligned}
& J(\boldsymbol{\Phi})=\sum_{p=1}^{P}\left(\boldsymbol{s}_{p}\right)^{\mathrm{H}} \boldsymbol{E}_{n} \boldsymbol{E}_{n}^{\mathrm{H}}\left(\boldsymbol{s}_{p}\right)-\kappa \\
& \quad \times \exp \left\{-\sum_{p=1}^{P}\left[\left(\boldsymbol{\Theta}_{s p}-\boldsymbol{\Theta}_{d p}\right)^{\mathrm{T}} \boldsymbol{D}_{p}^{-1}\left(\boldsymbol{\Theta}_{s p}-\boldsymbol{\Theta}_{d p}\right)\right] / 2\right\}
\end{aligned}
$$

where $\boldsymbol{s}_{p}=\left[\begin{array}{llll}s_{p 1} & s_{p 2} & \ldots & s_{p M}\end{array}\right]^{\mathrm{T}}=\left[\begin{array}{lll}\exp \left(\mathrm{j} \nu_{p 1}\right) & \exp \left(\mathrm{j} \nu_{p 2}\right)\end{array}\right.$ $\left.\ldots \quad \exp \left(\mathrm{j} \nu_{p M}\right)\right]^{\mathrm{T}}, \boldsymbol{\Theta}_{s p}=\left[\begin{array}{llll}\nu_{p 1} & \nu_{p 2} & \ldots & \nu_{p M}\end{array}\right]^{\mathrm{T}}$, and $\boldsymbol{\Phi}=$ $\left[\begin{array}{llll}\Theta_{s 1}^{\mathrm{T}} & \Theta_{s 2}^{\mathrm{T}} & \ldots & \Theta_{\mathrm{sP}}^{\mathrm{T}}\end{array}\right]^{\mathrm{T}}$. The first term of (13) represents the squared norm of the projection of the constraint vectors $\boldsymbol{s}_{p}, p=1,2, \ldots, P$ on the noise subspace spanned by $\boldsymbol{E}_{n}$. The second term is the likelihood function related constraint. $\kappa$ denotes a positive weighting parameter providing the relative weight between these terms. As a result, the optimal solution $\boldsymbol{\Phi}_{\mathrm{o}}$ of minimizing (13) can then be used as an appropriate estimate of $\boldsymbol{\Phi}_{P}=\left[\begin{array}{llll}\boldsymbol{\Theta}_{1}^{\mathrm{T}} & \boldsymbol{\Theta}_{2}^{\mathrm{T}} & \ldots & \boldsymbol{\Theta}_{P}^{\mathrm{T}}\end{array}\right]^{\mathrm{T}}$ formed by the actual phase angle vectors $\Theta_{p}, p=1,2, \ldots, P$ for array beamforming. However, the cost function of (13) is a highly nonlinear function of the phase angle vectors $\Theta_{s p}, p=1,2, \ldots, P$. Thus, a closed-form solution for the optimal solution cannot exist. We resort to an iterative procedure to solve this problem, as follows. First, we rewrite (13) as follows:

$$
\begin{aligned}
& J(\boldsymbol{\Phi})=(\mathbf{S})^{\mathrm{H}} \mathbf{W}(\mathbf{S})-\kappa \\
& \quad \times \exp \left\{-\sum_{p=1}^{P}\left[\left(\boldsymbol{\Theta}_{s p}-\boldsymbol{\Theta}_{d p}\right)^{\mathrm{T}} \boldsymbol{D}_{p}^{-1}\left(\boldsymbol{\Theta}_{s p}-\boldsymbol{\Theta}_{d p}\right)\right] / 2\right\}
\end{aligned}
$$


where the MP $\times 1$ vector $\mathbf{S}=\left[\begin{array}{llll}\boldsymbol{s}_{1}^{\mathrm{T}} & \boldsymbol{s}_{2}^{\mathrm{T}} & \ldots & \boldsymbol{s}_{P}^{\mathrm{T}}\end{array}\right]^{\mathrm{T}}$, and $\mathbf{W}$ is a $\mathrm{PM} \times \mathrm{PM}$ block diagonal matrix with the $p$ th $M \times M$ diagonal block matrix given by $\boldsymbol{E}_{n} \boldsymbol{E}_{n}^{\mathrm{H}}$. Then, the gradient vector of $J(\boldsymbol{\Phi})$ is computed according to

$$
\begin{aligned}
\nabla_{\Phi} J(\boldsymbol{\Phi})= & {\left[-2 \operatorname{Re}\left\{\mathrm{j}\left\{\left(\boldsymbol{E}_{n} \boldsymbol{E}_{n}^{\mathrm{H}} \boldsymbol{s}_{1}\right) \odot \boldsymbol{s}_{1}^{*}\right\}\right\}\right.} \\
& -2 \operatorname{Re}\left\{\mathrm{j}\left\{\left(\boldsymbol{E}_{n} \boldsymbol{E}_{n}^{\mathrm{H}} \boldsymbol{s}_{2}\right) \odot \boldsymbol{s}_{2}^{*}\right\}\right\} \ldots \\
& \left.-2 \operatorname{Re}\left\{\mathrm{j}\left\{\left(\boldsymbol{E}_{n} \boldsymbol{E}_{n}^{\mathrm{H}} \boldsymbol{s}_{P}\right) \odot \boldsymbol{s}_{P}^{*}\right\}\right\}\right]^{\mathrm{T}} \\
& +\kappa\left[\operatorname { e x p } \left\{-\sum_{p=1}^{P}\left[\left(\boldsymbol{\Theta}_{s p}-\boldsymbol{\Theta}_{d p}\right)^{\mathrm{T}} \boldsymbol{D}_{p}^{-1}\right.\right.\right. \\
& \left.\left.\left.\times\left(\boldsymbol{\Theta}_{s p}-\boldsymbol{\Theta}_{d p}\right)\right] / 2\right\} \boldsymbol{\Omega}^{-1}\left(\boldsymbol{\Phi}-\boldsymbol{\Phi}_{d}\right)\right]
\end{aligned}
$$

where $\operatorname{Re}\{\mathrm{x}\}$ denotes the real part of $\mathrm{x}$, the superscript * the complex conjugate, and $\odot$ the Hadamard (or elementwise) product [23]. $\boldsymbol{\Phi}_{d}=$ $\left[\begin{array}{llll}\Theta_{d 1}^{\mathrm{T}} & \Theta_{d 2}^{\mathrm{T}} & \ldots & \Theta_{\mathrm{dP}}^{\mathrm{T}}\end{array}\right]^{\mathrm{T}}$. Then, we update the phase angle vector $\boldsymbol{\Phi}$ and the corresponding steering constraint vector $s_{p}$ as follows:

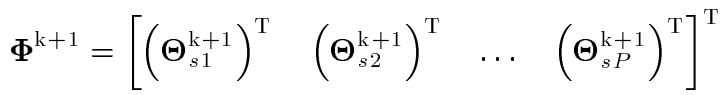

$$
\begin{aligned}
& =\boldsymbol{\Phi}^{\mathrm{k}}-\varepsilon \nabla_{\Phi} J\left(\boldsymbol{\Phi}^{\mathrm{k}}\right) \\
& s_{p m}^{\mathrm{k}+1}=\exp \left(\mathrm{j} \nu_{p m}^{\mathrm{k}+1}\right), \quad p=1,2, \ldots, P \\
& m=1,2, \ldots, M
\end{aligned}
$$

where the superscript $\mathrm{k}$ denotes the $\mathrm{k}$ th iteration and $\varepsilon$ the preset positive step size. From (16), we note that the second term includes the factor of likelihood function related to each of the phase angle vector errors $\boldsymbol{\Theta}_{s p}-\boldsymbol{\Theta}_{d p}, p=1,2, \ldots, P$ at the kth iteration. Hence, it would be expected that the resulting gradient approach for finding the optimal solution of $\boldsymbol{\Phi}$ can provide a more appropriate estimate of $\boldsymbol{\Phi}$ since the resulting step size becomes variable according to the exponential term, as shown in (15). In the literature, Smith [24] uses a conjugate gradient and Newton's method to maximize the array output signal-to-interference plus noise ratio (SINR) by adjusting the phase terms of the so-called phase-only weights for achieving the optimal phase-only adaptive nulling.

Next, we present an appropriate scheme for choosing the initial guess for the MP $\times 1$ vector $\mathbf{S}=\left[\begin{array}{llll}\boldsymbol{s}_{1}^{\mathrm{T}} & \boldsymbol{s}_{2}^{\mathrm{T}} & \ldots & \boldsymbol{s}_{P}^{\mathrm{T}}\end{array}\right]^{\mathrm{T}}$ in order to initiate the iterative process of the proposed robust method. According to the optimal weight vector given by (5) under the assumption of $P=1$ and the desired signal with direction vector $\boldsymbol{a}\left(\theta_{p}\right)$, the output of the adaptive array is approximately given by

$$
\mathrm{y}_{p}(\mathrm{t})=\boldsymbol{w}_{\mathrm{op}}^{H} \boldsymbol{x}(t) \approx s_{p}(\mathrm{t}) g_{p}+\boldsymbol{w}_{\mathrm{op}}^{\mathrm{H}} \boldsymbol{n}(\mathrm{t})
$$

based on the assumptions that $M>K$ and the interference signals are suppressed enough, where $g_{p} \equiv \boldsymbol{w}_{\mathrm{op}}^{\mathrm{H}} \boldsymbol{a}\left(\theta_{p}\right)$ denotes the array gain for the specified signal, where subscript $p$ represents the results obtained by using the desired signal with direction vector $\boldsymbol{a}\left(\theta_{p}\right), p=1,2, \ldots, P$. Equation (18) reveals that the output of the adaptive array can be used as a reference signal to find the actual phase angle vector $\Theta_{p}$. Consider the cross-correlation between $\boldsymbol{x}(\mathrm{t})$ and $\mathrm{y}_{p}(\mathrm{t})$. We have

$$
\begin{aligned}
E\left\{\boldsymbol{x}(\mathrm{t}) \mathrm{y}_{p}(\mathrm{t})^{*}\right\} & =E\left\{\boldsymbol{x}(\mathrm{t}) \boldsymbol{x}(\mathrm{t})^{\mathrm{H}}\right\} \boldsymbol{w}_{\mathrm{op}} \\
& =\boldsymbol{R}_{x} \boldsymbol{w}_{\mathrm{op}} \approx \pi_{p} g_{p}^{*} \boldsymbol{a}\left(\theta_{p}\right)+\sigma_{n}^{2} \boldsymbol{w}_{\mathrm{op}}
\end{aligned}
$$

where $\pi_{p}$ denotes the power associated with the specified signal. In practice, the noise power $\sigma_{n}^{2}$ is unknown. However, it can be obtained by setting the smallest eigenvalue of the autocorrelation matrix as the estimate of $\sigma_{n}^{2}$. From (19), we can therefore adopt the following vector as the initial guess for each of $\boldsymbol{s}_{p}$ :

$$
\boldsymbol{v}_{p}=\boldsymbol{R}_{x} \boldsymbol{w}_{\mathrm{op}}-\sigma_{n}^{2} \boldsymbol{w}_{\mathrm{op}} .
$$

Based on (20), it is clear that the direction vector $\boldsymbol{a}\left(\theta_{p}\right)$ is approximately proportional to $\boldsymbol{v}_{p}$ with a proportional constant $\pi_{p} g_{p}^{*}$. Hence, an appropriate initial guess $\boldsymbol{s}_{p}^{\circ}$ for $\boldsymbol{s}_{p}$ can be constructed as follows:

$$
\begin{aligned}
\boldsymbol{u}_{p} & =\left[u_{p 1}, u_{p 2}, \ldots, u_{p M}\right]^{\mathrm{T}}=\left(v_{p 1}\right)^{-1} \boldsymbol{v}_{p} \\
s_{p m}^{0} & =\left|u_{p m}\right|^{-1} u_{p m}, \quad m=1,2, \ldots, M \\
\boldsymbol{s}_{p}^{\circ} & =\left[s_{p 1}^{0}, s_{p 2}^{0}, \ldots, s_{p M}^{0}\right]^{\mathrm{T}}
\end{aligned}
$$

for $p=1,2, \ldots, P$, where $v_{p 1}$ denotes the first entry of $\boldsymbol{v}_{p}$ and $\boldsymbol{v}_{p}$ the result given by (20). The superscript "o" represents the initial guess. In other words, we keep only the phase portion of $v_{p}$ and then take the phase referencing to the first element of $\boldsymbol{v}_{p}$ to form the initial guess $\boldsymbol{s}_{p}^{\circ}$.

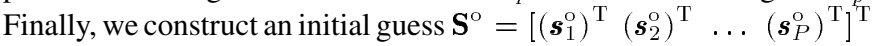
of $\mathbf{S}$ for carrying out the proposed iterative process.

For practical implementation, we compute the sample data correlation matrix $\underline{\boldsymbol{R}}_{\mathrm{x}}(i)$ using $i$ data snapshots as follows: $\underline{\boldsymbol{R}}_{\mathrm{x}}(i)=(1-$ $1 / i) \underline{\boldsymbol{R}}_{\mathrm{x}}(i-1)+(1 / i) \boldsymbol{x}(i) \boldsymbol{x}(i)^{\mathrm{H}}$, which is used as the estimate of the required $\boldsymbol{R}_{x}$, where $\boldsymbol{x}(i)$ denotes the $i$ th data snapshot sampled from $\boldsymbol{x}(\mathrm{t})$. The required computational complexity is $O\left(M^{2}\right)$. Using the approach of [22] to perform the eigendecomposition of $\underline{\boldsymbol{R}}_{\mathrm{x}}(i)$ provides the basis matrix $\underline{E}_{\mathrm{n}}(i)$ and needs $O\left(M^{2}\right)$. To construct the matrix $\underline{\boldsymbol{E}}_{\mathrm{n}}(i) \underline{\boldsymbol{E}}_{\mathrm{n}}(i)^{\mathrm{H}}$ requires $O\left(M^{3}\right)$. Therefore, the required computational complexity is about $O\left(M^{3}\right)+O\left(M^{2}\right)+\mathrm{PM}^{2}+\mathrm{PM}$ in order to obtain the first term of the right-hand side of (15). Moreover, the computational complexity for obtaining the second term of the right-hand side of (15) is about $3 \mathrm{MP}+M$. As a result, the computational complexity required for computing the gradient of $J(\boldsymbol{\Phi})$ when receiving $i$ data snapshots is about $O\left(M^{3}\right)+O\left(M^{2}\right)+\mathrm{PM}^{2}+4 \mathrm{MP}+M$.

\section{Convergence of the Proposed Method}

To ensure the convergence of the proposed method, we have to show that the cost function to be minimized as given by (14) possesses the property of $J\left(\boldsymbol{\Phi}^{\mathrm{k}+1}\right)<J\left(\boldsymbol{\Phi}^{\mathrm{k}}\right)$. Let the vector $\boldsymbol{A}^{\mathrm{k}}$ represent the second term of (16), i.e., $\boldsymbol{A}^{\mathrm{k}}=-\varepsilon \nabla_{\Phi} J\left(\boldsymbol{\Phi}^{\mathrm{k}}\right)=$ $\left[\begin{array}{llll}\left(\boldsymbol{A}_{1}^{\mathrm{k}}\right)^{\mathrm{T}} & \left(\boldsymbol{A}_{2}^{\mathrm{k}}\right)^{\mathrm{T}} & \ldots & \left(\boldsymbol{A}_{P}^{\mathrm{k}}\right)^{\mathrm{T}}\end{array}\right]^{\mathrm{T}}$ and $\boldsymbol{A}_{p}^{\mathrm{k}}=\left[\begin{array}{llll}A_{p 1}^{\mathrm{k}} & A_{p 2}^{\mathrm{k}} & \ldots & A_{p M}^{\mathrm{k}}\end{array}\right]^{\mathrm{T}}, p=$ $1,2, \ldots, P$. Assume that $\boldsymbol{A}^{\mathrm{k}}$ is a nonzero real vector with a norm small enough. Then, $\left(\boldsymbol{A}^{\mathrm{k}}\right)^{\mathrm{T}} \boldsymbol{A}^{\mathrm{k}}>0$, i.e.,

$$
\begin{aligned}
& \left(\boldsymbol{A}^{\mathrm{k}}\right)^{\mathrm{T}}\left\{2 \varepsilon \operatorname{Re}\left\{\mathrm{j}\left[\mathbf{W}\left(\mathbf{S}^{\mathrm{k}}\right)\right] \odot\left(\mathbf{S}^{\mathrm{k}}\right)^{*}\right\}-\varepsilon \kappa\right. \\
& \quad \times \exp \left\{-\left[\left(\boldsymbol{\Phi}^{\mathrm{k}}-\boldsymbol{\Phi}_{d}\right)^{\mathrm{T}} \boldsymbol{\Omega}^{-1}\left(\boldsymbol{\Phi}^{\mathrm{k}}-\boldsymbol{\Phi}_{d}\right)\right] / 2\right\} \\
& \left.\quad \times \boldsymbol{\Omega}^{-1}\left(\boldsymbol{\Phi}^{\mathrm{k}}-\boldsymbol{\Phi}_{d}\right)\right\}>0 .
\end{aligned}
$$

Hence

$$
\begin{aligned}
& \left(\boldsymbol{A}^{\mathrm{k}}\right)^{\mathrm{T}}\left\{2 \operatorname{Re}\left\{\mathrm{j}\left[\mathbf{W}\left(\mathbf{S}^{\mathrm{k}}\right)\right] \odot\left(\mathbf{S}^{\mathrm{k}}\right)^{*}\right\}\right\}>\kappa \\
& \quad \times \exp \left\{-\left[\left(\boldsymbol{\Phi}^{\mathrm{k}}-\boldsymbol{\Phi}_{d}\right)^{\mathrm{T}} \boldsymbol{\Omega}^{-1}\left(\boldsymbol{\Phi}^{\mathrm{k}}-\boldsymbol{\Phi}_{d}\right)\right] / 2\right\} \\
& \quad \times\left(\boldsymbol{A}^{\mathrm{k}}\right)^{\mathrm{T}} \boldsymbol{\Omega}^{-1}\left(\boldsymbol{\Phi}^{\mathrm{k}}-\boldsymbol{\Phi}_{d}\right)
\end{aligned}
$$

and the objective function after the $(\mathrm{k}+1)$ th iteration is given by

$$
\begin{aligned}
J\left(\boldsymbol{\Phi}^{\mathrm{k}+1}\right)= & \left(\mathbf{S}^{\mathrm{k}+1}\right)^{\mathrm{H}} \mathbf{W}\left(\mathbf{S}^{\mathrm{k}+1}\right)-\kappa \\
& \times \exp \left\{-\left[\left(\boldsymbol{\Phi}^{\mathrm{k}+1}-\boldsymbol{\Phi}_{d}\right)^{\mathrm{T}} \boldsymbol{\Omega}^{-1}\left(\boldsymbol{\Phi}^{\mathrm{k}+1}-\boldsymbol{\Phi}_{d}\right)\right] / 2\right\} .
\end{aligned}
$$

According to the above definition, (16), and (17), we have

$$
\begin{aligned}
& s_{p m}^{\mathrm{k}+1}=\exp \left(\mathrm{j} \nu_{p m}^{\mathrm{k}+1}\right)=\exp \left\{\mathrm{j}\left(\nu_{p m}^{\mathrm{k}}+\mathrm{A}_{p m}^{\mathrm{k}}\right)\right\} \\
& \approx\left(1+\mathrm{j} \mathrm{A}_{p m}^{\mathrm{k}}\right) \exp \left(\mathrm{j} \nu_{p m}^{\mathrm{k}}\right) \\
& p=1,2, \ldots, P, \quad m=1,2, \ldots, M
\end{aligned}
$$

hence, $\mathbf{S}^{\mathrm{k}+1} \approx \mathbf{S}^{\mathrm{k}}+\left(\mathrm{j} \boldsymbol{A}^{\mathrm{k}}\right) \odot \mathbf{S}^{\mathrm{k}}$. 


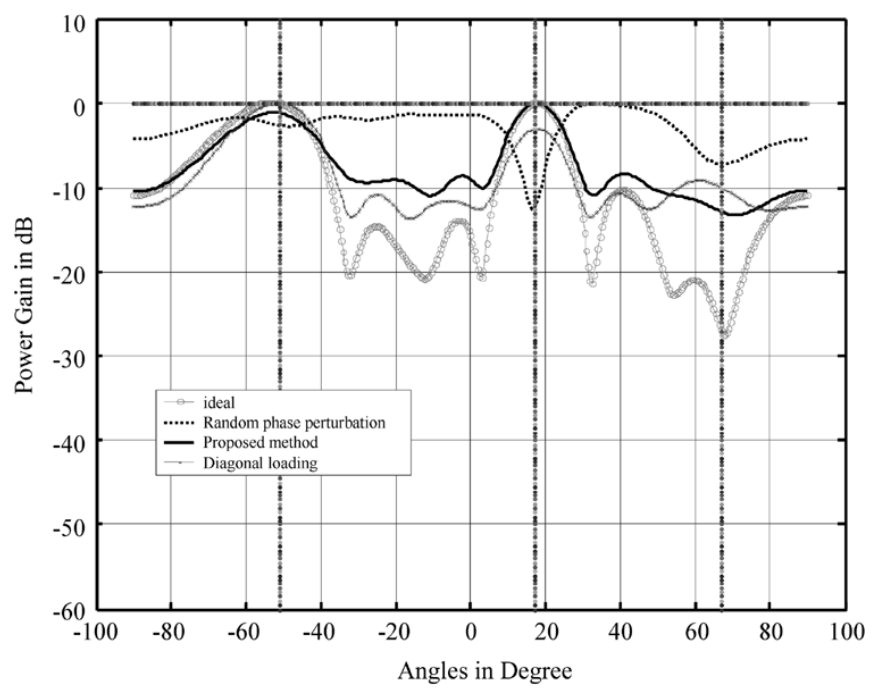

(a)

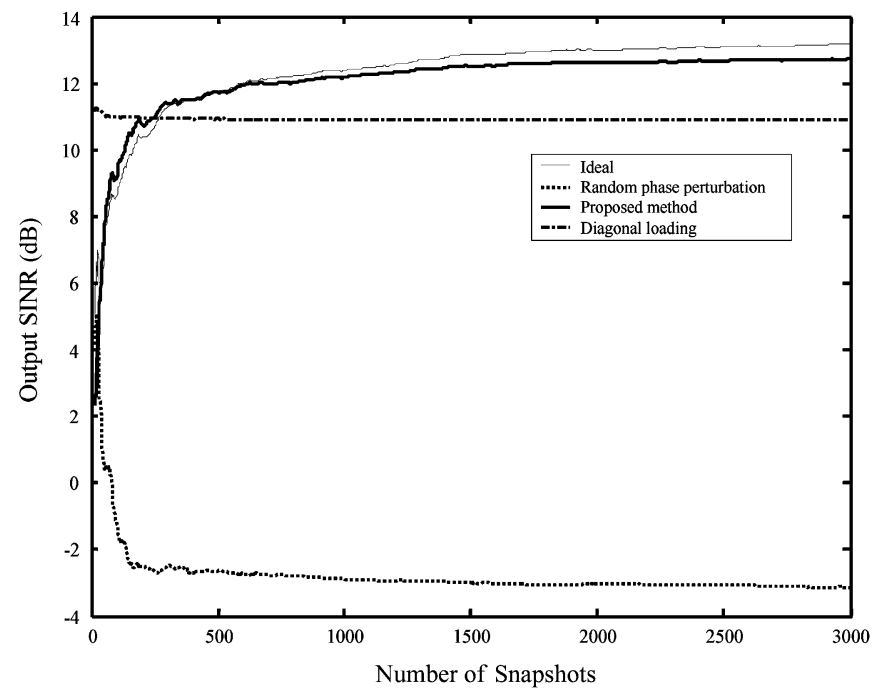

(c)

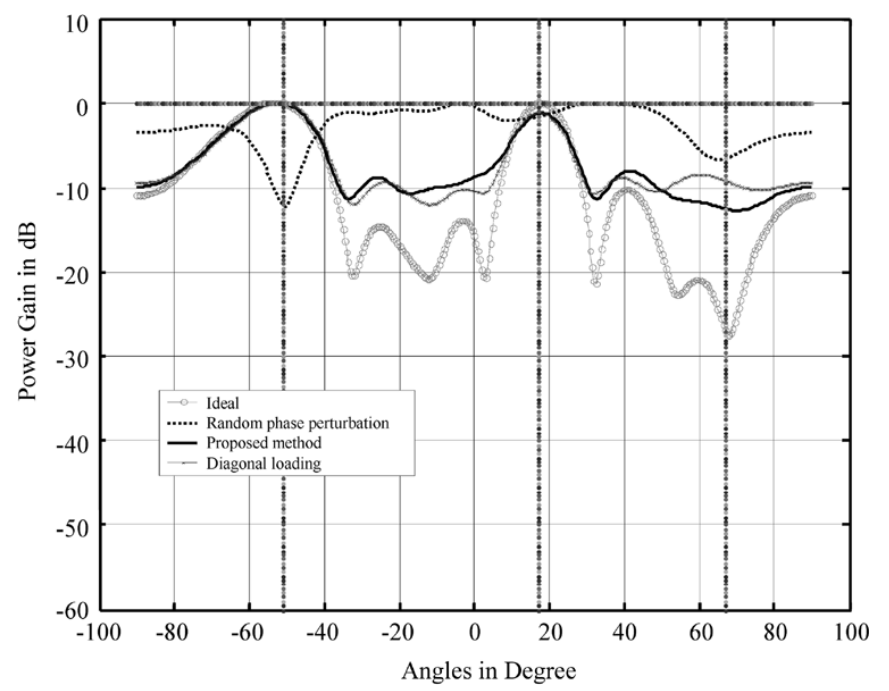

(b)

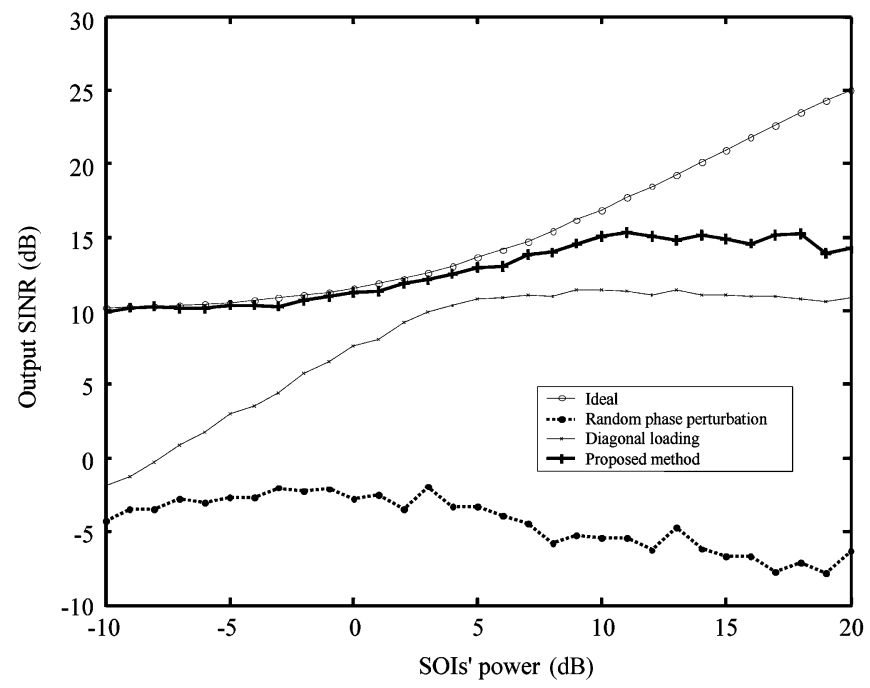

(d)

Fig. 1(a). Array beampattern with respect to the desired signal of (a) $17^{\circ}$, (b) $-51^{\circ}$, (c) output SINR versus the number of snapshots, and (d) array output SINR versus the SOIs' power, for Example 1.

Substituting (25) into (24) and performing the necessary algebraic manipulations yields

$$
\begin{aligned}
J\left(\boldsymbol{\Phi}^{\mathrm{k}+1}\right) & \\
\approx & {\left[\mathbf{S}^{\mathrm{k}}+\left(\mathrm{j} \boldsymbol{A}^{\mathrm{k}}\right) \odot \mathbf{S}^{\mathrm{k}}\right]^{\mathrm{H}} \mathbf{W}\left[\mathbf{S}^{\mathrm{k}}+\left(\mathrm{j} \boldsymbol{A}^{\mathrm{k}}\right) \odot \mathbf{S}^{\mathrm{k}}\right]-\kappa } \\
& \times \exp \left\{-\left[\left(\boldsymbol{\Phi}^{\mathrm{k}}+\boldsymbol{A}^{\mathrm{k}}-\boldsymbol{\Phi}_{d}\right)^{\mathrm{T}} \boldsymbol{\Omega}^{-1}\right.\right. \\
& \left.\left.\times\left(\boldsymbol{\Phi}^{\mathrm{k}}+\boldsymbol{A}^{\mathrm{k}}-\boldsymbol{\Phi}_{d}\right)\right] / 2\right\} \\
\approx & \left(\mathbf{S}^{\mathrm{k}}\right)^{\mathrm{H}} \mathbf{W}\left(\mathbf{S}^{\mathrm{k}}\right)-\kappa \\
\times & \exp \left\{-\left[\left(\boldsymbol{\Phi}^{\mathrm{k}}-\boldsymbol{\Phi}_{d}\right)^{\mathrm{T}} \boldsymbol{\Omega}^{-1}\left(\boldsymbol{\Phi}^{\mathrm{k}}-\boldsymbol{\Phi}_{d}\right)\right] / 2\right\} \\
& +\left(\mathbf{S}^{\mathrm{k}}\right)^{\mathrm{H}} \odot\left[-\mathrm{j} \boldsymbol{A}^{\mathrm{k}}\right] \mathbf{W}\left(\mathbf{S}^{\mathrm{k}}\right)+\left(\mathbf{S}^{\mathrm{k}}\right)^{\mathrm{H}} \mathbf{W}\left[\mathrm{j} \boldsymbol{A}^{\mathrm{k}}\right] \odot\left(\mathbf{S}^{k}\right) \\
& +\left(\mathbf{S}^{\mathrm{k}}\right)^{\mathrm{H}} \odot\left[-\mathrm{j} \boldsymbol{A}^{\mathrm{k}}\right] \mathbf{W}\left[\mathrm{j} \boldsymbol{A}^{\mathrm{k}}\right] \odot\left(\mathbf{S}^{\mathrm{k}}\right) \\
& +\left\{\left(\boldsymbol{\Phi}^{\mathrm{k}}-\boldsymbol{\Phi}_{d}\right)^{\mathrm{T}} \boldsymbol{\Omega}^{-1} \boldsymbol{A}^{\mathrm{k}}+\left(\boldsymbol{A}^{\mathrm{k}}\right)^{\mathrm{T}} \boldsymbol{\Omega}^{-1} \boldsymbol{A}^{\mathrm{k}} / 2\right\} \kappa \\
& \times \exp \left\{-\left[\left(\boldsymbol{\Phi}^{\mathrm{k}}-\boldsymbol{\Phi}_{d}\right)^{\mathrm{T}} \boldsymbol{\Omega}^{-1}\left(\boldsymbol{\Phi}^{\mathrm{k}}-\boldsymbol{\Phi}_{d}\right)\right] / 2\right\} \\
\approx & J\left(\boldsymbol{\Phi}^{\mathrm{k}}\right)+2 \mathrm{Re}\left\{\left(\mathbf{S}^{\mathrm{k}}\right)^{\mathrm{H}} \mathbf{W}\left[j \boldsymbol{A}^{\mathrm{k}}\right] \odot\left(\mathbf{S}^{\mathrm{k}}\right)\right\} \\
& +\left(\boldsymbol{\Phi}^{\mathrm{k}}-\boldsymbol{\Phi}_{d}\right)^{\mathrm{T}} \boldsymbol{\Omega}^{-1} \boldsymbol{A}^{\mathrm{k}} \boldsymbol{\kappa} \\
& \times \exp \left\{-\left[\left(\boldsymbol{\Phi}^{\mathrm{k}}-\boldsymbol{\Phi}_{d}\right)^{\mathrm{T}} \boldsymbol{\Omega}^{-1}\left(\boldsymbol{\Phi}^{\mathrm{k}}-\boldsymbol{\Phi}_{d}\right)\right] / 2\right\}
\end{aligned}
$$

since the norm of $\boldsymbol{A}^{\mathrm{k}}$ is small enough, and we neglect the terms $\left(\boldsymbol{A}^{\mathrm{k}}\right)^{\mathrm{T}} \boldsymbol{\Omega}^{-1} \boldsymbol{A}^{\mathrm{k}}$ and $\left(\mathbf{S}^{\mathrm{k}}\right)^{\mathrm{H}} \odot\left[-\mathrm{j} \boldsymbol{A}^{\mathrm{k}}\right] \mathbf{W}\left[\mathrm{j} \boldsymbol{A}^{\mathrm{k}}\right] \odot\left(\mathbf{S}^{\mathrm{k}}\right)$.

From (26), it is clear that we have to show

$$
\begin{aligned}
2 \operatorname{Re}\left\{\left(\mathbf{S}^{\mathrm{k}}\right)^{\mathrm{H}} \mathbf{W}\left[\mathbf{j} \boldsymbol{A}^{\mathrm{k}}\right] \odot\left(\mathbf{S}^{\mathrm{k}}\right)\right\}+\kappa\left[\left(\boldsymbol{\Phi}^{\mathrm{k}}-\boldsymbol{\Phi}_{d}\right)^{\mathrm{T}} \boldsymbol{\Omega}^{-1} \boldsymbol{A}^{\mathrm{k}}\right] \\
\exp \left\{-\left[\left(\boldsymbol{\Phi}^{\mathrm{k}}-\boldsymbol{\Phi}_{d}\right)^{\mathrm{T}} \boldsymbol{\Omega}^{-1}\left(\boldsymbol{\Phi}^{\mathrm{k}}-\boldsymbol{\Phi}_{d}\right)\right] / 2\right\} \leqq 0
\end{aligned}
$$

for any $\mathrm{k}$ to ensure the convergence. Based on (23), the condition of (27) can be reformulated as follows:

$$
\begin{aligned}
2 \operatorname{Re}\left\{\left(\mathbf{S}^{\mathrm{k}}\right)^{\mathrm{H}} \mathbf{W}\left[\mathrm{j} \boldsymbol{A}^{\mathrm{k}}\right] \odot\left(\mathbf{S}^{\mathrm{k}}\right)\right\} & \\
& +\left(\boldsymbol{A}^{\mathrm{k}}\right)^{\mathrm{T}} 2 \operatorname{Re}\left\{\mathrm{j}\left[\mathbf{W} \mathbf{S}^{\mathrm{k}}\right] \odot\left(\mathbf{S}^{\mathrm{k}}\right)^{*}\right\} \leqq 0
\end{aligned}
$$

which can be repressed as the following condition:

$$
\begin{aligned}
\sum_{p=1}^{P} 2 \operatorname{Re}\left\{\left(\mathbf{S}_{p}^{\mathrm{k}}\right)^{\mathrm{H}} \boldsymbol{E}_{n} \boldsymbol{E}_{n}^{\mathrm{H}}\left[\mathrm{j} \boldsymbol{A}_{p}^{\mathrm{k}}\right] \odot\left(\mathbf{S}_{p}^{\mathrm{k}}\right)\right\} \\
+\sum_{p=1}^{P}\left(\boldsymbol{A}_{p}^{\mathrm{k}}\right)^{\mathrm{T}} 2 \operatorname{Re}\left\{\left[\mathrm{j} \boldsymbol{E}_{n} \boldsymbol{E}_{n}^{\mathrm{H}} \mathbf{S}_{p}^{\mathrm{k}}\right] \odot\left(\mathbf{S}_{p}^{\mathrm{k}}\right)^{*}\right\} \leqq 0 .
\end{aligned}
$$




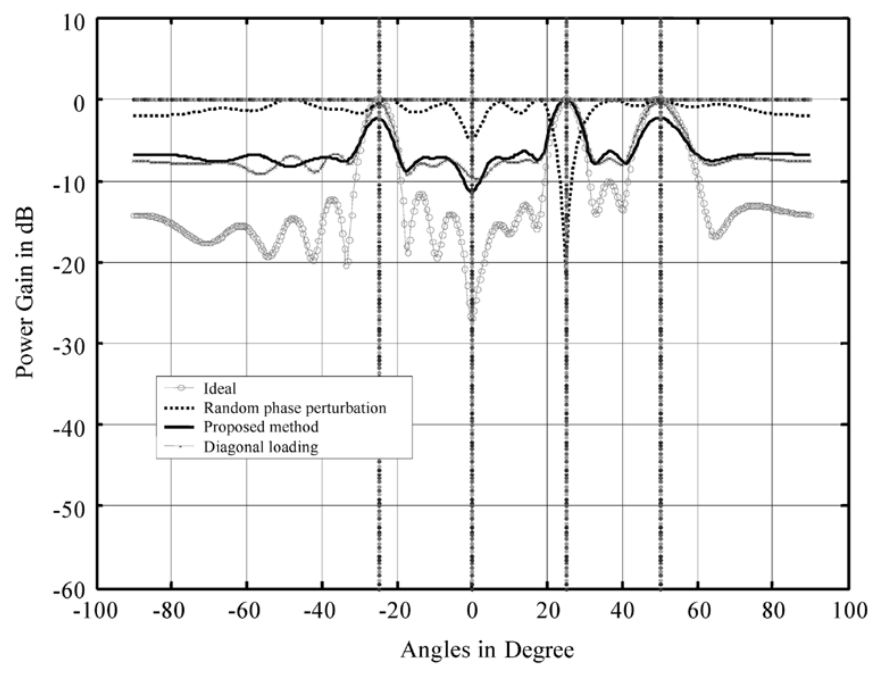

(a)

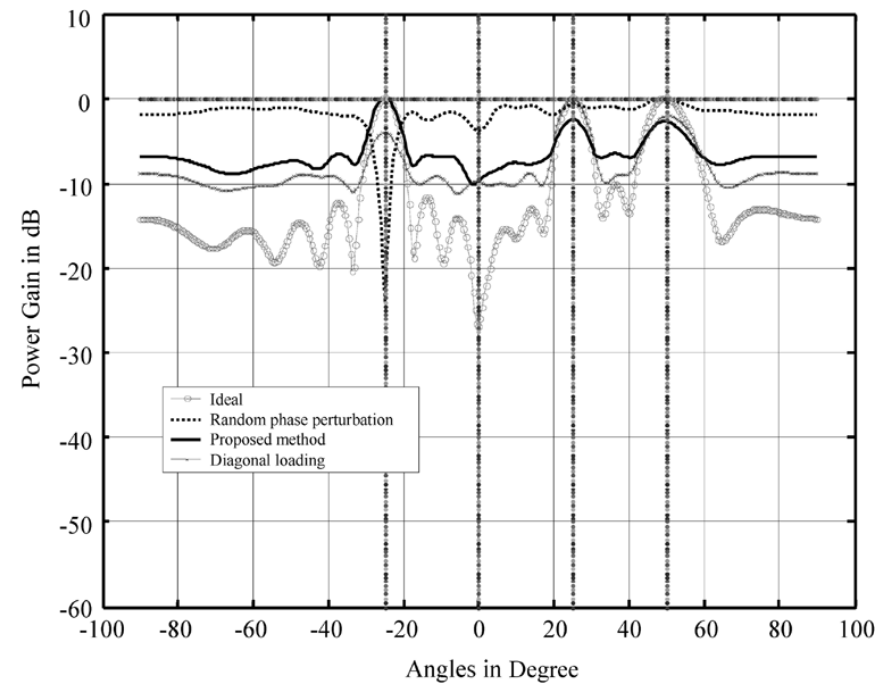

(b)

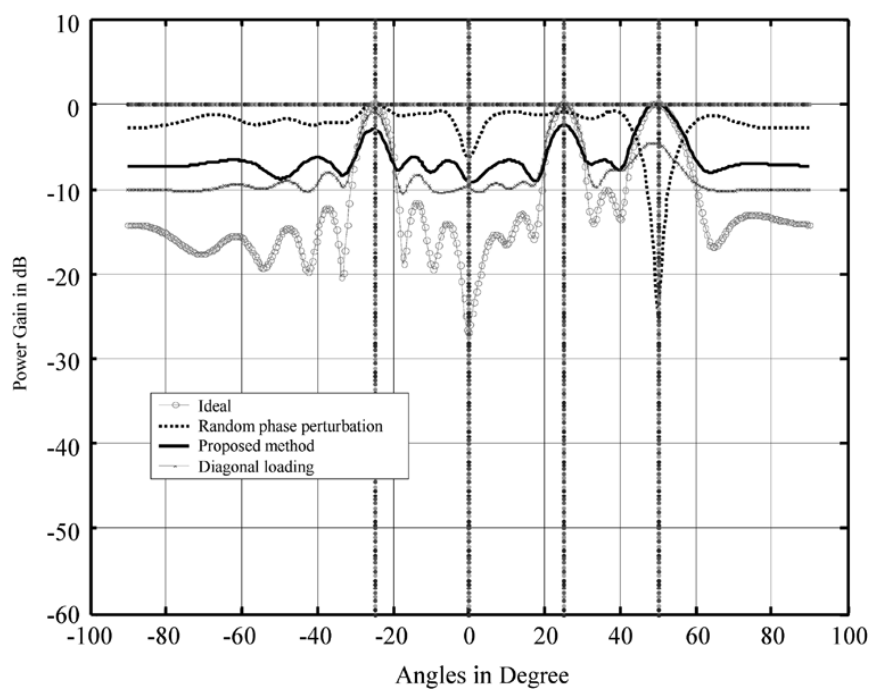

(c)

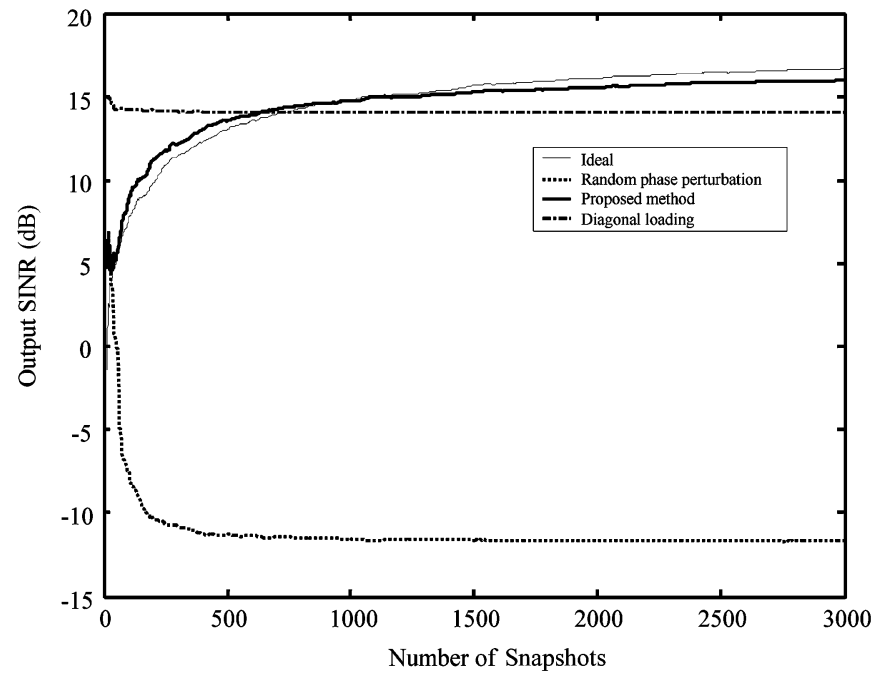

(d)

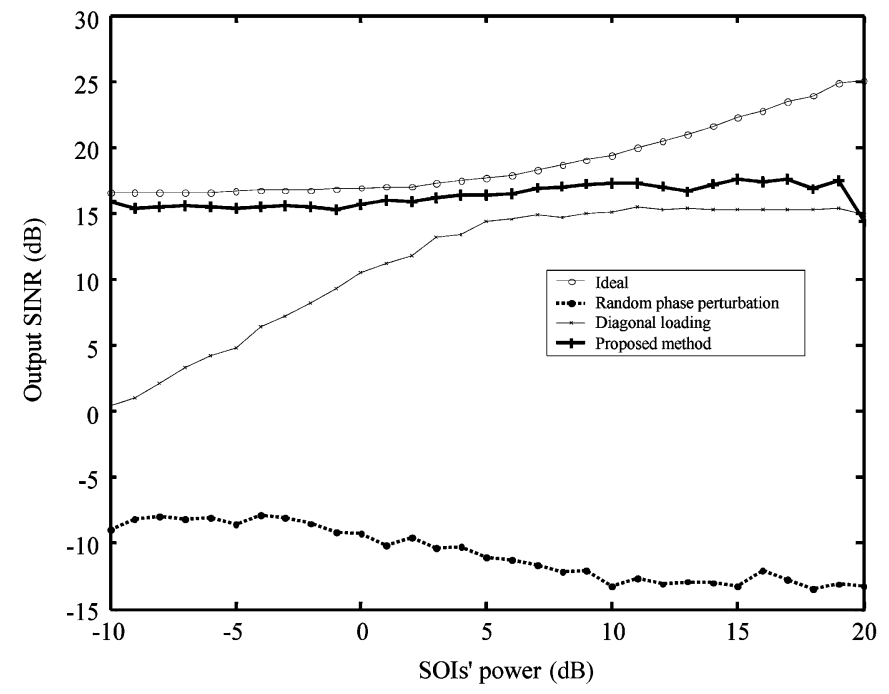

(e)

Fig. 2. Array beampattern with respect to the desired signal of (a) $25^{\circ}$, (b) $-25^{\circ}$, (c) $50^{\circ}$, (d) output SINR versus the number of snapshots, and (e) array output SINR versus the SOIs' power for Example 2. 
Next, the left-hand side of (29) is equal to

$$
\begin{aligned}
& \sum_{p=1}^{P} 2 \operatorname{Re}\left\{\left(\mathbf{S}_{p}^{\mathrm{k}}\right)^{\mathrm{H}} \boldsymbol{E}_{n} \boldsymbol{E}_{n}^{\mathrm{H}}\left[\mathrm{j} \boldsymbol{A}_{p}^{\mathrm{k}}\right] \odot\left(\mathbf{S}_{p}^{\mathrm{k}}\right)\right. \\
&\left.+\left(\boldsymbol{A}_{p}^{\mathrm{k}}\right)^{\mathrm{T}} \mathrm{j}\left[\boldsymbol{E}_{n} \boldsymbol{E}_{n}^{\mathrm{H}} \mathbf{S}_{p}^{\mathrm{k}}\right] \odot\left(\mathbf{S}_{p}^{\mathrm{k}}\right)^{*}\right\} \\
&=\sum_{p=1}^{P} 2 \operatorname{Re}\left\{\left(\mathbf{S}_{p}^{\mathrm{k}}\right)^{\mathrm{H}} \boldsymbol{E}_{n} \boldsymbol{E}_{n}^{\mathrm{H}}\left[\mathrm{j} \boldsymbol{A}_{p}^{\mathrm{k}}\right] \odot\left(\mathbf{S}_{p}^{\mathrm{k}}\right)\right. \\
&\left.+\left[\boldsymbol{E}_{n} \boldsymbol{E}_{n}^{\mathrm{H}} \mathbf{S}_{p}^{\mathrm{k}}\right]^{\mathrm{T}}\left[\mathrm{j} \boldsymbol{A}_{p}^{\mathrm{k}}\right] \odot\left(\mathbf{S}_{p}^{\mathrm{k}}\right)^{*}\right\} \\
&=\sum_{p=1}^{P} 2 \operatorname{Re}\left\{\left(\mathbf{S}_{p}^{\mathrm{k}}\right)^{\mathrm{H}} \boldsymbol{E}_{n} \boldsymbol{E}_{n}^{\mathrm{H}}\left[\mathrm{j} \boldsymbol{A}_{p}^{\mathrm{k}}\right] \odot\left(\mathbf{S}_{p}^{\mathrm{k}}\right)\right. \\
&\left.-\left\{\left(\mathbf{S}_{p}^{\mathrm{k}}\right)^{\mathrm{H}}\left[\boldsymbol{E}_{n} \boldsymbol{E}_{n}^{\mathrm{H}}\right]^{\mathrm{H}}\left[\mathrm{j} \boldsymbol{A}_{p}^{\mathrm{k}}\right] \odot\left(\mathbf{S}_{p}^{\mathrm{k}}\right)\right\}\right\} \\
&= \sum_{p=1}^{P} 2 \operatorname{Re}\left\{2 \mathrm{j} \operatorname{Im}\left\{\left(\mathbf{S}_{p}^{\mathrm{k}}\right)^{\mathrm{H}} \boldsymbol{E}_{n} \boldsymbol{E}_{n}^{\mathrm{H}}\left[\mathrm{j} \boldsymbol{A}_{p}^{\mathrm{k}}\right] \odot\left(\mathbf{S}_{p}^{\mathrm{k}}\right)\right\}\right\}=0 .
\end{aligned}
$$

Hence, the result given by the left-hand side of (28) is always equal to zero, i.e.,

$$
\begin{aligned}
2 \operatorname{Re}\left\{\left(\mathbf{S}^{\mathrm{k}}\right)^{\mathrm{H}} \mathbf{W}\left[\mathrm{j} \boldsymbol{A}^{\mathrm{k}}\right] \odot\right. & \left.\left(\mathbf{S}^{\mathrm{k}}\right)\right\} \\
& +\left(\boldsymbol{A}^{\mathrm{k}}\right)^{\mathrm{T}} 2 \operatorname{Re}\left\{\mathrm{j}\left[\mathbf{W} \mathbf{S}^{\mathrm{k}}\right] \odot\left(\mathbf{S}^{\mathrm{k}}\right)^{*}\right\}=0 .
\end{aligned}
$$

Consequently, we obtain

$$
\begin{aligned}
2 \operatorname{Re}\left\{\left(\mathbf{S}^{\mathrm{k}}\right)^{\mathrm{H}}\right. & \left.\mathbf{W}\left[\mathrm{j} \boldsymbol{A}^{\mathrm{k}}\right] \odot\left(\mathbf{S}^{\mathrm{k}}\right)\right\}+\left(\boldsymbol{\Phi}^{\mathrm{k}}-\boldsymbol{\Phi}_{d}\right)^{\mathrm{T}} \boldsymbol{\Omega}^{-1} \boldsymbol{A}^{k} \kappa \\
& \times \exp \left\{-\left[\left(\boldsymbol{\Phi}^{\mathrm{k}}-\boldsymbol{\Phi}_{d}\right)^{\mathrm{T}} \boldsymbol{\Omega}^{-1}\left(\boldsymbol{\Phi}^{\mathrm{k}}-\boldsymbol{\Phi}_{d}\right)\right] / 2\right\}<0 .
\end{aligned}
$$

It follows from (26) and (32) that $J\left(\boldsymbol{\Phi}^{\mathrm{k}+1}\right)<J\left(\boldsymbol{\Phi}^{\mathrm{k}}\right)$. This ensures the convergence property under suitably small selections of $\varepsilon$.

\section{COMPUter Simulation Results}

A ULA with interelement spacing equal to half of the minimum wavelength of the signals with specified gain/null requirements is used. All results presented are obtained by averaging $L$ independent runs with independent noise samples for each run. The noise power level for each of the simulation example is set to 1 . The array beampattern with respect to the desired signal of $\theta^{\circ}$ represents a beampattern obtained by using the searching vector $\boldsymbol{s}$ with phase angle equal to the presumed phase angle plus the phase angle error associated with the desired signal of $\theta^{\circ}$ for finding the power gain $\left|\boldsymbol{w}^{\mathrm{H}} \boldsymbol{s}\right|^{2}$.

Example 1: Three signal sources with signal-to-noise (SNR) equal to 5,4 , and $2 \mathrm{~dB}$, respectively, are impinging on the array with size $M=8$ from direction angels $17^{\circ},-51^{\circ}$, and $67^{\circ}$, respectively. The specified signals are the first two signals with $c_{1}=c_{2}=1$, and the third one is the jammer. Let the random phase angle error vector for each of the specified signals have zero mean and covariance matrix $\boldsymbol{D}_{p}=0.3 \boldsymbol{I}, p=1,2$, where $\boldsymbol{I}$ is the $8 \times 8$ identity matrix. Fig. 1 plots the simulation results in terms of the array beampatterns using 15000 data snapshots and the corresponding array output SINR with and without utilizing the proposed method. The values of $L, \varepsilon$, and $\kappa$ used are 50, 0.05, and 0.001, respectively. The results without random phase angle errors and of using the method of [17] with loading value equal to 2000 are also shown. The output SINRs obtained by utilizing 15000 data snapshots for the result of using the proposed method, the result without random phase angle errors, and the result of using the diagonal loading [17] are $13.06 \mathrm{~dB}, 13.58 \mathrm{~dB}$, and $10.92 \mathrm{~dB}$, respectively. We observe from this figure that the proposed method can effectively cope with the performance degradation due to the random phase angle errors.

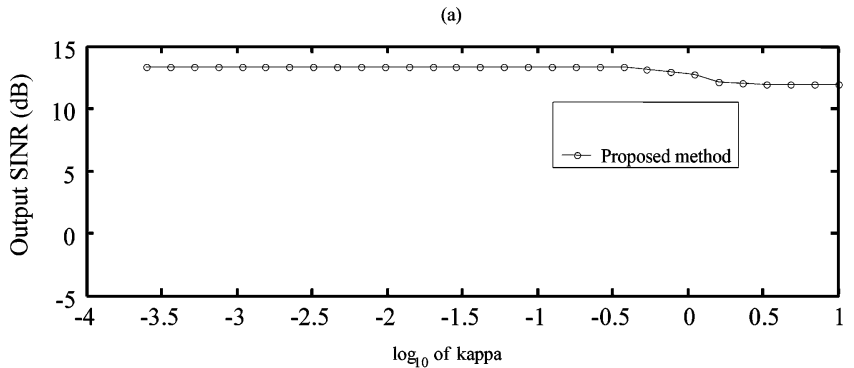

(b)

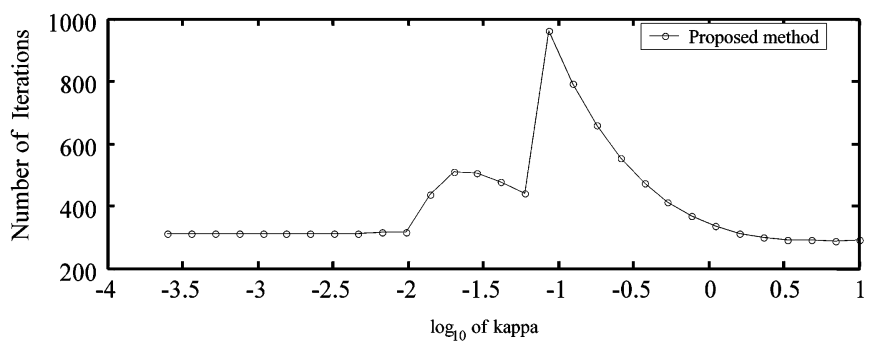

Fig. 3. Output SINR and number of iterations versus the relative weight for Example 1.

(a)

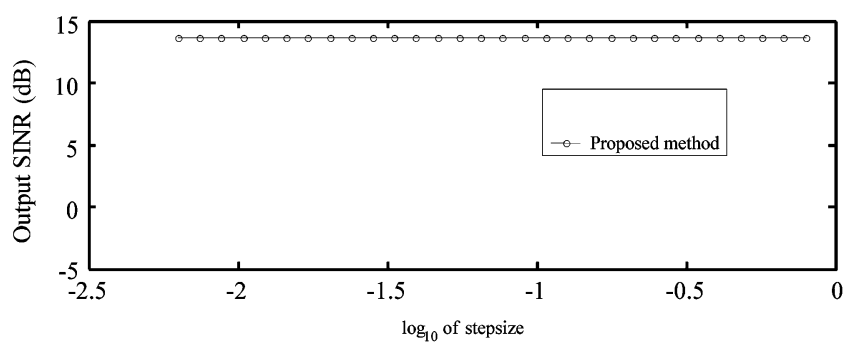

(b)

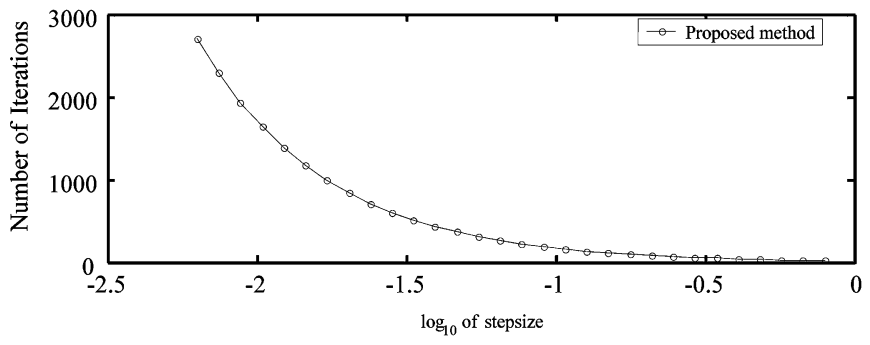

Fig. 4. Output SINR and number of iterations versus the step size for Example 1.

Example 2: Four signals with $\mathrm{SNR}$ equal to 5, 6, 7, and $5 \mathrm{~dB}$, respectively, are impinging on the array with size $M=15$ from direction angels $25^{\circ},-25^{\circ}, 50^{\circ}$, and $0^{\circ}$, respectively. The specified signals are the first three signals with $c_{1}=c_{2}=c_{3}=1$, and the fourth one is the jammer. Let the random phase angle error vector for each of the specified signals have zero mean and covariance matrix $\boldsymbol{D}_{p}=0.5 \boldsymbol{I}, p=1,2,3$, where $\boldsymbol{I}$ is the $15 \times 15$ identity matrix. Fig. 2 depicts the simulation results in terms of the array beampatterns using 15000 data snapshots and the corresponding array output SINR with and without utilizing the proposed method. The values of $L, \varepsilon$, and $\kappa$ used are 50,0.05, and 0.001, respectively. The results without random phase angle errors and of using the method of [17] with loading value equal to 2000 are also shown. The output SINRs obtained by utilizing 15000 data snapshots for the result of using the proposed method, the result without random phase angle errors, and the result of using the diagonal loading [17] 
are $17.48,17.75$, and $14.11 \mathrm{~dB}$, respectively. Again, we observe from this figure that the proposed method can effectively cure the performance degradation due to the random phase angle errors.

Finally, Figs. 3 and 4 show the array output SINRs and the numbers of iterations versus $\kappa$ and $\varepsilon$, respectively, for Example 1, after using 15000 data snapshots and taking the average of 20 independent runs. The proposed method provides array performance with robust capabilities not very sensitive to the choice of $\kappa$ and $\varepsilon$ in the ranges shown by the figures. However, the number of iterations for obtaining the convergent results is sensitive to the choice of $\kappa$ and $\varepsilon$ and decreases in general as the value of $\varepsilon$ increases.

\section{CONCLUSION}

We have illustrated that the performance degradation of an adaptive beamformer with multiple-beam constraints due to random phase angle errors is significant and presented an efficient method for solving the problem. A new cost function for curing the problem has been developed. The resulting minimization problem is highly nonlinear but can be solved through the use of an iterative procedure. In conjunction with a steepest-descent algorithm, the estimates for all of the signals with specified gain constraints can be obtained simultaneously. The convergence property regarding the proposed method has been investigated. Simulation examples have shown the effectiveness of the proposed method.

\section{REFERENCES}

[1] A. M. Vural, "Effects of perturbation on the performance of optimum adaptive arrays," IEEE Trans. Aerosp. Electron. Syst., vol. AES-15, pp. 76-87, Feb. 1979.

[2] R. T. Compton Jr., "Pointing accuracy and dynamic range in a steered beam adaptive array," IEEE Trans. Aerosp. Electron. Syst., vol. AES-16, pp. 280-287, May 1980.

[3] , "The effect of random steering vector errors in the Applebaum adaptive array," IEEE Trans. Aerosp. Electron. Syst., vol. AES-18, pp. 292-400, Sep. 1982.

[4] L. C. Godara, "Error analysis of the optimal antenna array processor," IEEE Trans. Aerosp. Electron. Syst., vol. AES-22, pp. 395-409, Mar. 1986.

[5] N. K. Jablon, "Adaptive beamforming with the generalized sidelobe canceller in the presence of array imperfections," IEEE Trans. Antennas Propag., vol. AP-34, pp. 996-1012, Aug. 1986.

[6] K. M. Ahmed and R. J. Evans, "Robust signal and array processing," in Proc. Inst. Elect. Eng. F-Commun. Radar Signal Process., Aug. 1982, pp. 297-302.

[7] R. J. Evans and K. M. Ahmed, "Robust adaptive array antennas," $J$. Acoust. Soc. Amer, vol. 71, pp. 384-394, Feb. 1982.

[8] M. H. Er and A. Cantoni, "An alternative formulation for an optimum beamformer with robust capability," in Proc. Inst. Elect. Eng. F-Commun. Radar Signal Process., Oct. 1985, pp. 447-460.

[9] M. H. Er, "A robust formulation for an optimum beamformer subject to amplitude and phase perturbation," Signal Process., vol. 19, pp. 17-26, Jan. 1990.

[10] M. H. Er and A. Cantoni, "Derivative constraints for broad-band element space antenna array processors," IEEE Trans. Acoust., Speech, Signal Process., vol. ASSP-31, pp. 1378-1393, Dec. 1983.

[11] K. M. Buckley and L. J. Griffiths, "An adaptive generalized sidelobe canceller with derivative constraints," IEEE Trans. Antennas Propag., vol. AP-34, pp. 311-319, Mar. 1986.

[12] H. Cox, R. M. Zeskind, and M. M. Owen, "Robust adaptive beamforming," IEEE Trans. Acoust., Speech, Signal Process., vol. ASSP-35, pp. 1365-1376, Oct. 1987.

[13] K. Takao and N. Kikuma, "Tamed adaptive antenna array," IEEE Trans. Aerosp. Electron. Syst., vol. AES-22, pp. 388-394, Mar. 1986.

[14] J. W. Kim and C. K. Un, "A robust adaptive array based on signal subspace approach," IEEE Trans. Signal Process., vol. 41, pp. 3166-3171, Nov. 1993

[15] A. Cantoni, X. G. Lin, and K. L. Teo, "A new approach to the optimization of robust antenna array processors," IEEE Trans. Antennas Propag., vol. 41, pp. 403-411, Apr. 1993.
[16] S. Shahbazpanahi, A. B. Gershman, Z.-Q. Luo, and K. M. Wong, "Robust adaptive beamforming for general-rank signal models using worstcase performance optimization," in Proc. 2nd IEEE Workshop Sonar Array Multichannel Process., Aug. 2002, pp. 13-17.

[17] J. Li, P. Stoica, and Z. Wang, "On robust Capon beamforming and diagonal loading," IEEE Trans. Signal Process., vol. 51, pp. 1702-1715, Jul. 2003.

[18] C.-C. Lee and J.-H. Lee, "Robust adaptive array beamforming under steering vector errors," IEEE Trans. Antennas Propag., vol. 45, pp. 168-175, Jan. 1997.

[19] J. T. Mayhan, "Area coverage adaptive nulling from geosynchronous satellites: Phased arrays versus multiple-beam antennas," IEEE Trans. Antennas Propag., vol. AP-34, pp. 410-419, Mar. 1986.

[20] K.-B. Yu, "Adaptive beamforming for satellite communication with selective earth coverage and jammer nulling capability," IEEE Trans. Signal Process., vol. 44, pp. 3162-3166, Dec. 1996.

[21] J.-H. Lee and T.-F. Hsu, "Adaptive beamforming with multiple-beam constraints in the presence of coherent jammers," Signal Process., vol. 80, pp. 2475-2480, Nov. 2000.

[22] G. Mathew, V. U. Reddy, and S. Dasgupta, "Adaptive estimation of eigensubspace," IEEE Trans. Signal Process., vol. 43, pp. 401-411, Feb. 1995.

[23] R. A. Horn and C. R. Johnson, Matrix Analysis. Cambridge, U.K.: Cambridge Univ. Press, 1985

[24] S. T. Smith, "Optimum phase-only adaptive nulling," IEEE Trans. Signal Process., vol. 47, pp. 1835-1843, July 1999.

\section{Online Bayesian Activity Detection in DS/CDMA Networks}

Thanh Ngoc Bui, Vikram Krishnamurthy, and H. Vincent Poor

Abstract-An on-line Bayesian based multiple hypotheses Shiryayev Sequential Probability Ratio Test (SSPRT) for the detection/isolation of new active users in a multiuser code division multiple access (CDMA) environment is presented. This SSPRT algorithm makes use of a priori knowledge of the user activity parameter. Comparison by simulation between this SSPRT algorithm and the non-Bayesian Matrix cumulative sum (CUSUM) shows that when such information is available, the SSPRT algorithm that uses this information can achieve better performance than the non-Bayesian approach.

Index Terms-Activity detection, CDMA, multiuser detection, sequential probability ratio test.

\section{INTRODUCTION}

Multiuser detection (MUD) has been shown to be an important demodulation technique for use in direct sequence code division multiple access (DS/CDMA) systems. Though many MUD schemes have been proposed, their performance depends significantly on the assumptions made about the interference parameters (for example,

Manuscript received March 16, 2003; revised December 15, 2003. This work was supported by NSERC Canada, the British Columbia Advanced Systems Institute, the U.S. National Science Foundation under Grant ANI-03-38807, and the New Jersey Center for Pervasive Information Techonology. The associate editor coordinating the review of this paper and approving it for publication was Dr. Joseph Tabrikian.

T. N. Bui is with the Department of Electrical and Electronic Engineering, University of Melbourne, Victoria 3010 Australia.

V. Krishnamurthy is with the Department of Electrical and Computer Engineering, University of British Columbia, Vancouver, BC, V6T 1Z4 Canada (e-mail vikramk@ece.ubc.ca).

H. V. Poor is with the Department of Electrical Engineering, Princeton University, Princeton, NJ 08544 USA (e-mail poor@princeton.edu).

Digital Object Identifier 10.1109/TSP.2004.838944 